\title{
Towards personalised therapy for lymphangioleiomyomatosis: lessons from cancer
}

\author{
Souheil El-Chemaly ${ }^{1,2}$ and Elizabeth P. Henske ${ }^{1,2}$
}

Affiliations: 'Division of Pulmonary and Critical Care Medicine, Dept of Medicine, Brigham and Women's Hospital, Harvard Medical School, Boston, MA, USA. ${ }^{2}$ Both authors contributed equally.

Correspondence: E.P. Henske, Division of Pulmonary and Critical Care Medicine, Dept of Medicine, Brigham and Women's Hospital, One Blackfan Circle, Karp 6th Floor, Boston, MA 02115, USA.

E-mail: ehenskelapartners.org

ABSTRACT Lymphangioleiomyomatosis (LAM) is a rare cystic, destructive lung disease occurring almost exclusively in females. Bi-allelic inactivating tuberous sclerosis complex (TSC) gene mutations occur in LAM cells, resulting in activation of the mTORC1 pathway. Pivotal clinical trials have demonstrated that inhibition of mTORC1 with sirolimus can induce a partial response of TSC-associated tumours and decrease the rate of lung function decline in females with LAM. Many parallels have been identified between LAM pathogenesis and neoplasia. Here, we highlight three key nodes through which advances in cancer therapy can streamline future innovation in clinical LAM research with parallels to breast and prostate cancer. These include: 1) hormonally targeted therapies to achieve true disease-free complete remissions; 2) the use of vascular endothelial growth factor-D and other plasma biomarkers to streamline early-phase clinical trials; and 3) the utilisation of histological and molecular features of biopsy material to enable patient stratification and personalised therapies.

0 @ERSpublications

This review discusses key lessons from cancer that the LAM community can build on towards personalised therapy in LAM http://ow.ly/slJLR

\section{Introduction}

Lymphangioleiomyomatosis (LAM) is a rare cystic lung disease that can occur in females with germline tuberous sclerosis complex (TSC) 1 or TSC2 gene mutations (TSC-LAM), or in a sporadic form in females who do not have TSC. Sporadic LAM is a multi-system disease in which pulmonary LAM cells and lung destruction are often accompanied by renal angiomyolipomas and retroperitoneal lymphadenopathy. A series of fast-paced breakthroughs in our understanding of LAM pathogenesis and therapy began with the discovery of bi-allelic inactivating TSC2 gene mutations in LAM cells from females with sporadic LAM [1], representing the first molecular link between sporadic LAM and TSC-LAM [2]. Unexpectedly, identical TSC2 mutations were identified in LAM cells and angiomyolipoma cells from sporadic LAM patients, leading to the hypothesis that LAM cells could migrate or metastasise [1]. Consistent with this hypothesis, recurrent LAM after lung transplantation was found to carry the same TSC2 mutation as the patient's

Received: Dec 092013 | Accepted after revision: Dec 202013

Support statement: S. El-Chemaly was supported by the National Institutes of Health/National Heart, Lung, and Blood Institute (grant no. 1 R21 HL119902). Research in the Henske Lab (Brigham and Women's Hospital, Harvard Medical School, Boston, MA, USA) is supported by the LAM Foundation, the Tuberous Sclerosis Alliance, the Department of Defense Tuberous Sclerosis Research Program, the National Heart, Lung, and Blood Institute, the National Institute of Diabetes and Digestive and Kidney Diseases, and the Adler Foundation.

Conflict of interest: Disclosures can be found alongside the online version of this article at err.ersjournals.com

Provenance: Submitted article, peer reviewed.

Copyright OERS 2014. ERR articles are open access and distributed under the terms of the Creative Commons Attribution Non-Commercial Licence 3.0. 
"native" LAM pre-transplantation, suggesting that LAM cells had migrated or metastasised to the donor lung [3]. Most recently, it has been proposed that LAM should be reclassified as a neoplastic disorder [4].

Subsequent to the discovery of TSC2 gene mutations in sporadic LAM, the TSC protein complex was found to regulate the activity of the mammalian target of rapamycin (mTOR) complex 1 (mTORC1), and mTOR hyperactivation was demonstrated in LAM and angiomyolipoma cells [5]. mTORC1 is activated in the majority of human solid tumours, promoting angiogenesis and altering cellular metabolism; thus, highlighting another connector between LAM and neoplasia [4]. A pivotal randomised, double-blind clinical trial, the MILES (Multicenter International LAM Efficacy of Sirolimus) trial, which examined the rate of decline in forced expiratory volume in $1 \mathrm{~s}$ (FEV1) in females with moderately severe LAM, demonstrated that sirolimus can stabilise lung function in females with moderately severe LAM. 46\% of subjects who received sirolimus had an FEV1 at or above baseline at the end of first year of the study, while the remaining $54 \%$ had continued lung function decline over the study period [6]. Importantly, however, lung function declines when the drug is discontinued [6]. This is consistent with data from other trials of TSC-associated tumours in which regrowth is observed upon treatment discontinuation [7].

Similarly, complete responses to single-agent mTORC1 inhibitors are rarely observed in malignant solid tumours, with notable exceptions including a case of bladder cancer in which a sustained response occurred in a tumour that contained somatic mutation in the TSC1 gene, along with mutations in other genes including neurofibromin 2 (NF2) [8]. Therefore, in cells and in vivo, mTORC1 inhibitors typically induce a cytostatic response in TSC-associated tumours, LAM and sporadic malignancies, with a partial decrease in cell and tumour size.

In addition to the parallels between LAM and neoplasia highlighted above, other similarities have been identified and reviewed $[4,5]$. These include the ability of LAM cells to metastasise to the lungs and other sites, the induction of angiogenesis and lymphangiogenesis, their invasion of the lung and their metabolic switch toward aerobic glycolysis, or the "Warburg effect" [9]. Given these parallels, how can the LAM research community use advances in cancer therapy to guide future breakthroughs? Herein, we highlight three specific pathways that may prove informative.

\section{Targeting the hormonal axis in LAM: parallels with breast and prostate cancer}

Targeting the hormonal axis in LAM could achieve complete long-lasting remissions, paralleling breast and prostate cancer (table 1).

Surprisingly, the loss of TSC2 with activation of mTORC1 does not promote cell growth or proliferation in most in vitro and in vivo models, despite the fact that the loss of TSC2 in humans leads to LAM, angiomyolipomas and other proliferative lesions. Autophagy is kept at low levels in LAM cells by the continuous activation of mTORC1, which is a master regulator of autophagy via multiple mechanisms, including the nuclear localisation of transcription factor EB [10], phosphorylation of UNC-51-like kinase-1 [11], and transcriptional regulation of autophagy-dependent genes [12]. Autophagy appears to represent a "metabolic cliff" for TSC2-deficient cells, since further molecular or pharmacological inhibition of

\section{TABLE 1 Prognostic and therapeutic predictors: parallels between LAM and breast and prostate cancer}

Host factors
Younger age
African-American
Tumour factors
Stage
Histology score
Lymphovascular involvement
Receptor status
Circulating tumour cells
Genetic profiling
Serum marker of response to therapy

Breast cancer

Prostate cancer

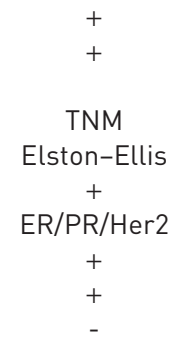

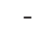

$+$

TNM

Gleason score

$+$

-

?

Decreased PSA
LAM

$+$

?

Lymphatic involvement?

LAM histology score

$+$

$\mathrm{ER} / \mathrm{PR} / \mathrm{BcL} 2 / \mathrm{TERT}$ ?

$+$

?

Decreased VEGF-D

LAM: Lymphangioleiomyomatosis; TNM: tumour, nodes, metastasis; ER: oestrogen receptor; PR: progesterone receptor; Her2: Human epidermal growth factor receptor-2; Bcl2: B-cell lymphoma-2; TERT: telomerase reverse transcriptase; PSA: prostate specific antigen; VEGF-D: vascular endothelial growth factor- $D_{;}+$: predictor associated with condition; -: predictor not associated with condition; ?: insufficient evidence regarding association. 
autophagy leads to cell death [13], and induction of autophagy is likely to contribute to the cytostatic response observed with rapalogue therapy. We hypothesise that mTORC1-dependent inhibition of autophagy limits the proliferative capacity of TSC2-deficient cells by inducing a "metabolic dormancy" state [13]. The hypothesis that the combination of mTORC1 inhibition plus autophagy inhibition has clinical benefit in LAM is currently being investigated in a phase I clinical trial, the SAIL (Sirolimus and Autophagy Inhibition in LAM) trial. Intriguingly, autophagy inhibition in TSC2-deficient cells leads to dependence on the pentose phosphate pathway, and the combination of chloroquine (to inhibit autophagy) and 6-aminonicotinamide (an antimetabolite that inhibits the pentose phosphate pathway) selectively inhibits the growth of TSC2-deficient but not TSC2-expressing cells [14].

Given that the loss of TSC2 appears to induce an autophagy-dependent metabolic dormancy-like state, why do LAM cells grow? Clearly, other factors are required, with oestrogen and other steroid hormones as top candidates. We hypothesise that the appropriate hormonal environment is required for LAM cells to exit their metabolic dormancy and enter a proliferative, destructive state. We propose a "two-hit model" of LAM pathogenesis, in which the loss of both alleles of TSC2 is the initiating event, but that the appropriate hormonal environment is essential to the progression of LAM. A key corollary of this hypothesis is that appropriate hormonal therapy will convert LAM cells from an active state back to a dormant state, with clinical benefit.

LAM cells and angiomyolipoma cells express oestrogen receptor (ER)- $\alpha$ and progesterone receptors [15-17]. LAM cells that are ER positive also express Bcl2 (B-cell lymphoma-2), an anti-apoptotic protein [18], suggesting a link between LAM cell survival and oestrogen pathways. Intriguingly, post-menopausal LAM patients treated with rapamycin have a greater loss of circulating LAM cells in the blood and urine than pre-menopausal females, again suggesting an important role for oestrogen in metastasis and survival of LAM cells [19].

In TSC2-deficient cells, 17- $\beta$ oestradiol increases cell size, and promotes cell survival, metastasis and lung colonisation via mitogen-activated protein kinase/extracellular signal-regulated kinase kinase (MEK)-dependent pathways [20-23]. Oestradiol also induces the expression and activity of matrix metalloproteinase-2 in TSC2-deficient cells, which may contribute to lung destruction [20, 24]. The prometastatic effects of oestradiol on TSC2-deficient cells can be blocked, in vitro and in vivo, by the US Food and Drug Administration approved agent fulvestrant (Faslodex; AstraZeneca, London, UK) [20], which leads to degradation of ER- $\alpha$.

In both breast and prostate cancer, hormonally targeted therapies can result in complete remission, something that has not yet been observed in LAM or angiomyolipomas treated with mTORC1 inhibitors. In breast cancer, the combination of an mTORC1 inhibitor with an aromatase inhibitor has proven efficacy [25]. In metastatic prostate cancer, disease that regrows despite first-line hormonal suppression with a gonadotropin releasing hormone agonist and an anti-androgen can respond to additional hormonal interventions, including new agents that target androgen synthesis, such as abiraterone [26] sometimes yielding a second complete remission.

Can hormonal pathways be targeted in LAM to achieve complete remissions? In vitro studies suggest that tamoxifen has oestrogen agonist effects in LAM [27]. Previous retrospective clinical analyses suggest that progesterone is not an effective therapy to halt lung function decline in females with LAM [28, 29]. A recent clinical trial, TRAIL (Trial of Aromatase Inhibition in LAM), has finished recruiting post-menopausal subjects with LAM who received treatment with letrozole, an aromatase inhibitor, and results should provide insights into the effects of hormonal therapy in LAM. Additional prospective trials are urgently needed, including trials in pre-menopausal females with LAM. Additional basic and translational research is also needed, including studies to determine whether LAM cells can synthesise intra-tumoural oestrogens and androgens, as can breast and prostate cancer cells.

In summary, the MILES trial showed that $46 \%$ of subjects who received sirolimus had an FEV1 at or above baseline at the end of the first study year, while the rest of the cohort had continued lung function decline over the study period [6]. Complete disease remissions have not been observed in TSC2-deficient angiomyolipomas or subependymal giant cell astrocytomas treated with rapalogues. Pre-clinical studies indicate that targeting hormonal stimuli in LAM with agents such as fulvestrant, which lack oestrogen agonist activity, could have therapeutic benefit [20]. In metastatic breast and prostate cancer, hormonally targeted therapy can lead to complete remission.

\section{Specific recommendation}

Prospectively controlled clinical trials of hormonally active agents, such as fulvestrant, or an aromatase inhibitor plus sirolimus are urgently needed in LAM. 
Plasma biomarkers could guide therapeutic decision-making: parallels with prostate-specific antigen and prostate cancer

In prostate cancer, prostate-specific antigen (PSA) has been used in phase I-II clinical trials as an indicator of therapeutic efficacy. Increasing PSA can be used as an indicator of disease progression, allowing intermittent androgen blockade in metastatic prostate cancer, with decreased adverse events and improved quality of life [30, 31]. Vascular endothelial growth factor (VEGF)-D has been shown to be a useful biomarker in the diagnosis of LAM [32], with elevated plasma levels $>800 \mathrm{pg} \cdot \mathrm{mL}^{-1}$ in approximately twothirds of females with biopsy-proven LAM. VEGF-D levels fell with sirolimus therapy and higher levels correlate with increased disease severity [33]. If it were possible to use VEGF-D and/or other biomarkers as surrogate indicators of therapeutic response in clinical trials of LAM, the duration, enrolment targets and costs of trials could drop significantly. For example, the recently published 2-year study of doxycycline in LAM showed that doxycycline does not impact change in lung function [34] or VEGF-D levels. Could the use of VEGF-D levels as surrogate end-points have resulted in a shorter trial? This would allow promising agents to be tested more quickly for preliminary signs of efficacy, thereby streamlining and prioritising agents for pivotal clinical trials using lung function as end-points. VEGF-D or other biomarker levels could also be used to monitor therapy, allowing rapamycin treatment breaks and thereby decreasing adverse events, as well as predicting when sirolimus therapy can be safely discontinued or the dose reduced in postmenopausal females.

\section{Specific recommendations}

Ongoing efforts to identify the source of the plasma VEGF-D in LAM and to correlate VEGF-D levels with clinical outcomes should be supported at the highest possible level. Additional plasma biomarkers should be sought with urgency because they are so critical to the pace of clinical trials in LAM. This includes biomarkers in the $30 \%$ of LAM patients who do not have elevated VEGF-D.

\section{Disease stratification based on histological and genomic features of LAM cells: parallels with breast and prostate cancer}

In LAM, clinical subsets based on noninvasive metrics are emerging: pre- versus post-menopause; high versus low VEGF-D levels; lymphatic involvement versus no lymphatic involvement [35, 36]; and rapid versus slow rate of lung function decline. A recent computational analysis of lung texture in areas around LAM lung cysts identified features that correlate with lung function decline [37]. Is tissue-based stratification needed for more precise phenotyping? Multiple histological studies have demonstrated significant cellular heterogeneity in LAM. The LAM histology score [38] is a validated predictor of the clinical course of LAM and is correlated with the intensity of lung tissue reactivity with VEGF receptor-3, an indicator of lymphatic endothelial cells [39]. In the context of obtaining tissue diagnosis, once prospective studies are performed, fibreoptic bronchoscopy could potentially be a useful, relatively safe tool to histologically diagnose LAM [40, 41].

In breast cancer, tissue-based analyses are used to classify disease subgroups (ER/progesterone receptor positive, human epidermal growth factor receptor-2 positive and triple negative), each of which has distinct treatment algorithms. A validated set of tissue-based analyses, the Oncotype Dx assay (Genomic Health Inc., Redwood City, CA, USA), is also widely used to predict the likelihood of disease recurrence and guide the use of adjuvant therapy. Obviously none of this would be possible if the tumours were not biopsied. Similarly, in prostate cancer, the Gleason score is used to guide therapeutic decisions and is predictive of the likelihood of disease recurrence.

In LAM, could knowledge of ER, progesterone receptor, telomerase reverse transcriptase and Bcl2 status, for example, be important in clinical decision making? Would information acquired from a biopsy justify the additional risks? Would this additional information help recruit specific patients to clinical trials and, therefore, increase the chances of successfully identifying novel therapies? If future clinical trials focus on the non-canonical functions of TSC and Rheb, outside the "canonical" mTORC1 signalling network [42, 43], will it be beneficial to know the status of these pathways in LAM tissue specimens? As we embark on an entirely new era of LAM therapy, in which many females will receive years of continuous, single-agent treatment with sirolimus, could tissue specimens identify females who would benefit from an additional agent targeting an mTORC1-dependent feedback loop?

\section{Specific recommendations}

The LAM community should thoughtfully consider the risks and potential benefits of tissue information before, during or after targeted therapy. Ultimately, biopsies could enable a personalised approach to therapy. 


\section{Conclusions}

The striking parallels with neoplasia, despite the fact that LAM cells have a histologically benign appearance, have already forged a unique alliance between pulmonary medicine and oncology within the LAM research and clinical care community. The progress in LAM in the past decade is remarkable. The development of a personalised approach with durable, complete remission is an achievable goal, with opportunities to use advances in cancer therapy to jump-start future clinical research advances in LAM. Two of the most hormonally sensitive malignancies, breast and prostate cancer, illustrate key nodes of potential synergy: 1) the development of hormonally targeted therapies to achieve complete remissions; 2) the use of plasma biomarkers to streamline clinical trials and individualise therapeutic decisions; and 3) the use of tissue-based molecular and cellular features to identify subgroups that require distinct therapeutic approaches. These research trajectories need to be carefully partnered with basic and clinical investigation into areas of LAM that are not closely paralleled by most malignancies, including lung destruction and lymphangiogenesis.

\section{References}

1 Carsillo T, Astrinidis A, Henske EP. Mutations in the tuberous sclerosis complex gene TSC2 are a cause of sporadic pulmonary lymphangioleiomyomatosis. Proc Natl Acad Sci USA 2000; 97: 6085-6090.

$2 \mathrm{Yu} \mathrm{J}$, Astrinidis A, Henske EP. Chromosome 16 loss of heterozygosity in tuberous sclerosis and sporadic lymphangiomyomatosis. Am J Respir Crit Care Med 2001; 164: 1537-1540.

3 Karbowniczek M, Astrinidis A, Balsara BR, et al. Recurrent lymphangiomyomatosis after transplantation: genetic analyses reveal a metastatic mechanism. Am J Respir Crit Care Med 2003; 167: 976-982.

4 McCormack FX, Travis WD, Colby TV, et al. Lymphangioleiomyomatosis: calling it what it is: a low-grade, destructive, metastasizing neoplasm. Am J Respir Crit Care Med 2012; 186: 1210-1212.

5 Henske EP, McCormack FX. Lymphangioleiomyomatosis - a wolf in sheep's clothing. J Clin Invest 2012; 122: 3807-3816.

6 McCormack FX, Inoue Y, Moss J, et al. Efficacy and safety of sirolimus in lymphangioleiomyomatosis. N Engl J Med 2011; 364: 1595-1606.

7 Bissler JJ, McCormack FX, Young LR, et al. Sirolimus for angiomyolipoma in tuberous sclerosis complex or lymphangioleiomyomatosis. N Engl J Med 2008; 358: 140-151.

8 Iyer G, Hanrahan AJ, Milowsky MI, et al. Genome sequencing identifies a basis for everolimus sensitivity. Science 2012; 338: 221.

9 Vander Heiden MG, Cantley LC, Thompson CB. Understanding the Warburg effect: the metabolic requirements of cell proliferation. Science 2009; 324: 1029-1033.

10 Martina JA, Chen Y, Gucek M, et al. MTORC1 functions as a transcriptional regulator of autophagy by preventing nuclear transport of TFEB. Autophagy 2012; 8: 903-914.

11 Jung $\mathrm{CH}$, Jun $\mathrm{CB}$, Ro $\mathrm{SH}$, et al. ULK-Atg13-FIP200 complexes mediate mTOR signaling to the autophagy machinery. Mol Biol Cell 2009; 20: 1992-2003.

12 Rosenbluth JM, Pietenpol JA. mTOR regulates autophagy-associated genes downstream of p73. Autophagy 2009; 5: 114-116.

13 Parkhitko A, Myachina F, Morrison TA, et al. Tumorigenesis in tuberous sclerosis complex is autophagy and p62/ sequestosome 1 (SQSTM1)-dependent. Proc Natl Acad Sci USA 2011; 108: 12455-12460.

14 Parkhitko AA, Priolo C, Coloff JL, et al. Autophagy-dependent metabolic reprogramming sensitizes TSC2-deficient cells to the antimetabolite 6-aminonicotinamide. Mol Cancer Res 2014; 12: 48-57.

15 Henske EP, Ao X, Short MP, et al. Frequent progesterone receptor immunoreactivity in tuberous sclerosisassociated renal angiomyolipomas. Mod Pathol 1998; 11: 665-668.

16 Logginidou $\mathrm{H}$, Ao X, Russo I, et al. Frequent estrogen and progesterone receptor immunoreactivity in renal angiomyolipomas from women with pulmonary lymphangioleiomyomatosis. Chest 2000; 117: 25-30.

17 Matsui K, Takeda K, Yu ZX, et al. Downregulation of estrogen and progesterone receptors in the abnormal smooth muscle cells in pulmonary lymphangioleiomyomatosis following therapy. An immunohistochemical study. Am J Respir Crit Care Med 2000; 161: 1002-1009.

18 Usuki J, Horiba K, Chu SC, et al. Immunohistochemical analysis of proteins of the Bcl-2 family in pulmonary lymphangioleiomyomatosis: association of Bcl-2 expression with hormone receptor status. Arch Pathol Lab Med 1998; 122: 895-902.

19 Cai X, Pacheco-Rodriguez G, Haughey M, et al. Sirolimus decreases circulating lymphangioleiomyomatosis cells in patients with lymphangioleiomyomatosis. Chest 2014; 145: 108-112.

20 Li C, Zhou X, Sun Y, et al. Faslodex inhibits estradiol-induced extracellular matrix dynamics and lung metastasis in a model of lymphangioleiomyomatosis. Am J Respir Cell Mol Biol 2013; 49: 135-142.

$21 \mathrm{Gu} \mathrm{X}, \mathrm{Yu}$ JJ, Ilter D, et al. Integration of mTOR and estrogen-ERK2 signaling in lymphangioleiomyomatosis pathogenesis. Proc Natl Acad Sci USA 2013; 110: 14960-14965.

22 Yu JJ, Robb VA, Morrison TA, et al. Estrogen promotes the survival and pulmonary metastasis of tuberin-null cells. Proc Natl Acad Sci USA 2009; 106: 2635-2640.

23 Shim B, Pacheco-Rodriguez G, Kato J, et al. Sex-specific lung diseases: effect of oestrogen on cultured cells and in animal models. Eur Respir Rev 2013; 22: 302-311.

24 Glassberg MK, Elliot SJ, Fritz J, et al. Activation of the estrogen receptor contributes to the progression of pulmonary lymphangioleiomyomatosis via matrix metalloproteinase-induced cell invasiveness. J Clin Endocrinol Metab 2008; 93: 1625-1633.

25 Baselga J, Campone M, Piccart M, et al. Everolimus in postmenopausal hormone-receptor-positive advanced breast cancer. N Engl J Med 2012; 366: 520-529.

26 de Bono JS, Logothetis CJ, Molina A, et al. Abiraterone and increased survival in metastatic prostate cancer. $N$ Engl J Med 2011; 364: 1995-2005. 

and activate both genomic and nongenomic signaling pathways. Am J Physiol Lung Cell Mol Physiol 2004; 286: L694-L700.

28 Taveira-Da Silva AM, Stylianou MP, Hedin CJ, et al. Decline in lung function in patients with lymphangioleiomyomatosis treated with or without progesterone. Chest 2004; 126: 1867-1874.

29 Johnson SR, Whale CI, Hubbard RB, et al. Survival and disease progression in UK patients with lymphangioleiomyomatosis. Thorax 2004; 59: 800-803.

30 Crook JM, O'Callaghan CJ, Duncan G, et al. Intermittent androgen suppression for rising PSA level after radiotherapy. N Engl J Med 2012; 367: 895-903.

31 Hussain M, Tangen CM, Berry DL, et al. Intermittent versus continuous androgen deprivation in prostate cancer. N Engl J Med 2013; 368: 1314-1325.

32 Young LR, Vandyke R, Gulleman PM, et al. Serum vascular endothelial growth factor-D prospectively distinguishes lymphangioleiomyomatosis from other diseases. Chest 2010; 138: 674-681.

33 Young LR, Lee HS, Inoue Y, et al. Serum VEGF-D concentration as a biomarker of lymphangioleiomyomatosis severity and treatment response: a prospective analysis of the Multicenter International Lymphangioleiomyomatosis Efficacy of Sirolimus (MILES) trial. Lancet Respir Med 2013; 1: 445-452.

34 Chang WY, Cane JL, Kumaran M, et al. A two year randomised placebo controlled trial of doxycycline for lymphangioleiomyomatosis. Eur Respir J 2013 [In press DOI: 10.1183/09031936.00167413].

35 Glasgow CG, Taveira-Da Silva A, Pacheco-Rodriguez G, et al. Involvement of lymphatics in lymphangioleiomyomatosis. Lymphat Res Biol 2009; 7: 221-228.

36 Glasgow CG, El-Chemaly S, Moss J. Lymphatics in lymphangioleiomyomatosis and idiopathic pulmonary fibrosis. Eur Respir Rev 2012; 21: 196-206.

37 Yao J, Taveira-Da Silva AM, Colby TV, et al. CT grading of lung disease in lymphangioleiomyomatosis. AJR Am J Roentgenol 2012; 199: 787-793.

38 Matsui K, Beasley MB, Nelson WK, et al. Prognostic significance of pulmonary lymphangioleiomyomatosis histologic score. Am J Surg Pathol 2001; 25: 479-484.

39 Kumasaka T, Seyama K, Mitani K, et al. Lymphangiogenesis in lymphangioleiomyomatosis: its implication in the progression of lymphangioleiomyomatosis. Am J Surg Pathol 2004; 28: 1007-1016.

40 Harari S, Torre O, Cassandro R, et al. Bronchoscopic diagnosis of Langerhans' cell histiocytosis and lymphangioleiomyomatosis. Respir Med 2012; 106: 1286-1292.

41 Meraj R, Wikenheiser-Brokamp KA, Young LR, et al. Utility of transbronchial biopsy in the diagnosis of lymphangioleiomyomatosis. Front Med 2012; 6: 395-405.

42 Atochina-Vasserman EN, Goncharov DA, Volgina AV, et al. Statins in lymphangioleiomyomatosis. Simvastatin and atorvastatin induce differential effects on tuberous sclerosis complex 2-null cell growth and signaling. Am J Respir Cell Mol Biol 2013; 49: 704-709.

43 Goncharova EA, Goncharov DA, Li H, et al. mTORC2 is required for proliferation and survival of TSC2-null cells. Mol Cell Biol 2011; 31: 2484-2498. 